\section{Resumen}

Este documento aporta elementos para la comprensión de un proyecto institucional, de educación superior inclusiva, de la Universidad Pedagógica Nacional, y su propósito gira en torno a la formación de licenciados sordos para atender las necesidades educativas de la población sorda colombiana. Manos y Pensamiento: Inclusión de estudiantes sordos a la vida universitaria, como se le denomina, es una propuesta en desarrollo en la que han estado presentes, además de la comunidad sorda, teóricos que nutren y proporcionan fundamentos para el debate sobre la constitución de sujeto y subjetividad del educando sordo, e investigadores en el tema de la atención a la diversidad, en escenarios de educación superior, que brindan valiosos elementos para la estructuración pedagógica del proyecto y el desarrollo de las distintas acciones que demanda. Ha sido pensado como un proyecto de gran complejidad, por los múltiples factores que debe abordar. No se trata de un simple proceso de vinculación universitaria, sino de un encuentro de culturas, lenguajes, mundos, formas de sentir, pensar e imaginar. Es el ejercicio permanente de la convivencia, bajo un mismo espacio y tiempo, de educandos en condiciones distintas que apenas empiezan a descubrirse y valorar lo que significan en un mundo social. Es, por tanto, una oportunidad de transformación social y ciudadana. El artículo, en tono propositivo, presenta los principales aspectos relacionados con el origen del proyecto y describe los referentes teóricos y legales que lo sustentan. Así mismo, narra el proceso seguido en la Universidad Pedagógica para la implementación de esta experiencia de inclusión educativa.

\section{Palabras clave}

Educación inclusiva, inclusión, diversidad, educación superior, maestros sordos

\section{Abstract}

This document contributes with some basic elements to the understanding of an institutional project on inclusive higher education at the Universidad Pedagogica Nacional. Its purpose is the training of specialized deaf teachers to cover educational needs of Colombian deaf population. Hands and thoughts: Inclusion of deaf students at University life, as it is known, is a developing proposal in which deaf population is involved as well as theory consultants, who enrich and give fundamentals to the debate about the building of subject and subjectivity of the deaf student, and researchers in the field of attention to diversity in higher education, which offer valuable facts for the pedagogical structure of the project and the development of the different actions which are required. This project has been thought as one of great complexity because of the multiple factors which must be considered. It is not only a simple process of university adaptation but also a mix of cultures, languages, citizenships, and ways of thought, feeling and imagination. It is the constant practice of coexistence in by the same time and on the same roof, of pupils in different conditions who just begin to discover themselves and appreciate what they mean in a social world. So, this is an opportunity for social and citizenship change. As a proposal this article presents the main aspects related to the origin of the project and describes the theoretical and legal referents which support it. On the other hand, it tells the process followed at the Universidad Pedagogica during this experience of inclusive education.

\section{Key words}

Inclusive education, higher education, inclusion, diversity, deaf teachers 


\title{
Inclusión de estudiantes sordos a la vida universitaria: Una experiencia significativa en la Universidad Pedagógica Nacional ${ }^{1}$
}

\author{
Nahir Rodríguez de Salazar ${ }^{2}$ \\ Diana Patricia García Ríos ${ }^{3}$ \\ María del Socorro Jutinico Fernández ${ }^{4}$
}

La Inclusión asume que vivir y aprender juntos es la mejor forma de beneficiar a cualquier persona Ainscow y Corbett $(1999)^{5}$

Existe en la actualidad una marcada tendencia hacia la inclusión social que demanda a los sistemas educativos, en los distintos países del mundo, la formación de hombres con capacidad para valorar la diferencia y reconocer la diversidad en sus distintas dimensiones.

Contrario a lo que acontecía hasta la década de los setenta, hoy la Educación Especial ha podido evolucionar de la segregación a la integración y, de ella, al paradigma de la educación inclusiva. En esta última transición, la universidad se ha visto abocada a replantear su compromiso con la sociedad y con todos los hombres que la constituyen, independientemente de su condición; ello no signi-

\footnotetext{
${ }^{1}$ Artículo recibido el 29 de febrero, evaluado el 7 de mayo y el 3 de junio, y arbitrado el 9 de junio de 2008.

${ }^{2}$ Magíster en Psicología Clínica. Profesora de la Facultad de Educación de la Universidad Pedagógica Nacional, Proyecto curricular de Educación con énfasis en Educación Especial. Coordinadora del Proyecto Manos y Pensamiento: Inclusión de estudiantes sordos a la vida universitaria. nahirs@pedagogica.edu.co

${ }^{3}$ Magíster en Educación Comunitaria. Profesora de la Facultad de Educación de la Universidad Pedagógica Nacional, Proyecto curricular de Educación con énfasis en Educación Especial. Integrante y coinvestigadora del Proyecto Manos y Pensamiento: Inclusión de estudiantes sordos a la vida universitaria. Integrante del grupo de investigación Equidad y Diversidad en Educación, Departamento de posgrados. dgarcia@pedagogica.edu.co

${ }^{4}$ Especialista en Educación con énfasis en Comunicación Aumentativa y Alternativa. Profesora de la Facultad de Educación de la Universidad Pedagógica Nacional, Proyecto curricular de Educación con énfasis en Educación Especial. Integrante y coinvestigadora del Proyecto Manos y Pensamiento: Inclusión de estudiantes sordos a la vida universitaria. mjutinico@pedagogica.edu.co

${ }^{5}$ Tomado del libro de Moriña, A. (2004, p. 15).
} 
fica que haya dejado de cumplir su papel en la generación de conocimiento y en la formación de investigadores e intelectuales; por el contrario, el movimiento de educación inclusiva le invita a reconocer otros sujetos, otras inteligencias, otras capacidades, otras formas de expresión a través de lenguajes distintos a los convencionalmente utilizados por grupos mayoritarios.

La idea de construir un mundo más tolerante y menos discriminatorio frente a la diversidad humana es una constante en los distintos planteamientos teóricos y experienciales revisados en el reciente Estado del arte $^{6}$, elaborado por el equipo de investigación Manos y Pensamiento. Sin duda, la educación es la institución social que mayor compromiso tiene en la reducción de esas profundas brechas que han polarizado los normales de los mal denominados anormales. La universidad no escapa a esta nueva recomprensión de sujeto con discapacidad; ella, por su función formadora, investigativa y de proyección social, es la instancia educativa con mayor compromiso en las trasformaciones sociales y culturales, y en la generación de redes interuniversitarias.

Nuestro propósito es presentar el camino recorrido por la Universidad Pedagógica Nacional (UPN) para re-comprender, en contexto educativo, fundamentos y acciones afirmativas desarrolladas a lo largo de esta experiencia pedagógica.

\section{¿Cómo nace el proyecto Manos y Pensamiento?}

Hace más de una década, algunos padres de familia con hijos sordos visitaron la Universidad Pedagógica, para solicitar atención a los procesos educativos de sus hijos. No teníamos, en ese momento, fundamentos teóricos suficientes para responder a su demanda. Dudábamos si la educación de las personas sordas nos correspondía toda vez que su problemática era, por tradición, objeto de intervención rehabilitatoria por parte del campo de la fonoaudiología. En el interior de la Facultad de Educación, hubo posturas a favor y en contra en torno al papel de la Universidad Pedagógica con ese tipo de población, y se recordó la existencia de antecedentes sobre un programa de formación de educadoras especiales con énfasis en sordos que, desafortunadamente, se ofreció por una sola vez debido a que no se surtieron los trámites legales requeridos para su aprobación.

La solicitud de los padres y algunas organizaciones de personas sordas fue pilar para considerar la introducción de una asignatura -Lenguaje manual- que se ofreció como electiva a estudiantes del Programa de Educación Especial. Años más tarde, el nombre de ésta fue sustituido por Lengua manual, debido a los desarrollos alcanzados en los estudios lingüísticos sobre personas sordas, realizados especialmente en Suecia, que evidenciaban que la lengua manual es un idioma y, por tanto, no debía confundirse con el concepto lenguaje como facultad humana.

\footnotetext{
${ }^{6}$ Proyecto investigativo finalizado, cuyo informe reposa en el Centro de Investigaciones de la Universidad Pedagógica Nacional, bajo el título Manos y Pensamiento: Proyecto educativo para la población sorda colombiana, desde la Universidad Pedagógica Nacional, Código DSI-O20-05.
} 
A medida que avanzaban los estudios lingüísticos, se generaron nuevos replanteamientos. Así empezó a cuestionarse hasta dónde era más válido hablar de Lengua de Señas debido a que, en este idioma visogestual, las manos no eran el único elemento. Teniendo estos argumentos como soporte, se hizo el cambio en la denominación de la asignatura que, desde entonces y hasta hoy, se ofrece como electiva desde la Facultad de Educación, a toda la comunidad universitaria.

Sin embargo, las nuevas visiones que se generaron por la dinámica de transformación nacional e internacional alrededor de la educación de la población sorda no se redujeron a la enseñanza de una lengua. Esto fue una invitación para iniciar, en la Universidad Pedagógica Nacional, procesos de intervención pedagógica, a través de diversos proyectos de grado desarrollados con instituciones para personas sordas, como el Instituto Nacional de Sordos (INSOR) y el Instituto Colombiano de Audición y Lenguaje (ICAL), hoy denominado Fundación para el niño sordo ICAL.

La participación de la Universidad Pedagógica en distintos encuentros, congresos y seminarios locales, regionales, nacionales e internacionales permitió, hacia finales de la década de los noventa, mayor comprensión sobre la necesidad de formar maestros para atender la educación de las personas sordas; como producto de estas reuniones quedó claro que, al igual que todo hombre, el sujeto sordo requiere, para desarrollarse humanamente, de una educación de calidad; por tanto, fue necesario superar los enfoques centrados en el desarrollo del lenguaje y avanzar hacia paradigmas pedagógicos que posibilitaran la comprensión de quién es la persona sorda, cómo construye conocimiento sobre el mundo y cuáles son los métodos y estrategias pedagógicas que permiten un mayor impacto en el desarrollo de su pensamiento, de su afectividad y demás dimensiones humanas.

En el año 2000, el Proyecto Curricular de Educación con énfasis en Educación Especial incorporó, dentro de sus áreas de interés, una en limitación auditi$v a$, como respuesta educativa a las necesidades sociales de la población sorda y sus familias.

Debe resaltarse que Colombia, país multiétnico y multicultural, requiere también de la formación de maestros sordos y maestras sordas para que se hagan partícipes activos del proceso educativo, en sus distintos niveles y modalidades. En el caso de la comunidad sorda, es prioritaria una formación profesoral que indague por su cultura, su lengua, sus formas de pensar y sus distintas expresiones y manifestaciones humanas, para borrar la brecha que nos separa, a los oyentes, de su mundo.

Hacia finales de los noventa, las instituciones de bachillerato gradúan los primeros sordos y, con ello, se genera una demanda educativa a las universidades, que tampoco estaban preparadas para responder a sus necesidades. Si bien, en la actualidad, la UPN ya cuenta con una importante experiencia en el tema de la educación bilingüe para las personas sordas, no cabe duda de que, ante la complejidad de su mundo, es necesario continuar fortaleciendo la formación y la 
investigación en temas relacionados con su capacidad cognitiva, las metodologías y didácticas para potenciar la lengua de señas y el castellano como segunda lengua, y la evaluación de los aprendizajes.

En abril de 2002, se presentó un anteproyecto a la UPN, a través de la Decanatura de la Facultad de Educación, que fue aprobado por el Consejo Académico como proyecto de investigación formativa, cuyo propósito era diseñar una propuesta de formación de profesores oyentes para atender la educación bilingüe y bicultural de personas sordas ${ }^{7}$. Para su desarrollo se proponía, en primera instancia, comprender las dimensiones del bilingüismo y del biculturalismo y sus implicaciones en la formación de docentes y, en segunda, diseñar una propuesta de formación de maestros para la comunidad sorda. Además, y como producto de los hallazgos, se proponía reorientar el área de interés en limitados auditivos, contemplada en el proyecto curricular de pregrado.

Es prudente resaltar que la propuesta de formar maestros para atender la educación bilingüe y bicultural en los distintos países del mundo tomó fuerza a partir de este mismo período.

De acuerdo con el panorama internacional en materia educativa, es necesario que estos maestros, además, obtengan una sólida formación en educación general y, de manera particular, comprendan la forma como los sordos conocen y se apropian del conocimiento, con el fin de generar adaptaciones curriculares pertinentes a las necesidades, sus intereses y contexto donde se proyecte la acción educativa de los sordos.

El desarrollo de este proyecto permitió la organización de un equipo de profesores de la UPN interesado en la educación bilingüe de la persona sorda, que fomentó encuentros con miembros de esta comunidad, padres de familia, asociaciones, colegios, universidades y organismos internacionales. El diálogo y la interacción con estos distintos actores tuvieron como propósito indagar sobre su historia educativa, el acceso a las lenguas, su nivel de aspiraciones y el tipo de tratamiento dado por las instituciones escolares.

El carácter narrativo de este trabajo de investigación participativa permitió conocer que la vida de los sordos colombianos no escapa de la planteada por Sánchez (1990), en su obra La increíble y triste historia de la sordera.

En nuestro país, los sordos también han sido objeto de rechazo social, aun dentro del proceso mismo de integración; los oyentes han usado términos peyorativos y les han comparado con comportamientos propios de animales.

Hasta el año 2002 el acceso a la vida universitaria era casi inexistente, solamente se encontraron no más de diez casos de personas sordas que tuvieron acceso a la educación superior, la mayoría en modalidad a distancia. En ningún caso las universidades financiaron el servicio de interpretación.

\footnotetext{
${ }^{7}$ Anteproyecto presentado, inicialmente, por Nahir Rodríguez, Sandra Guido y Esperanza Monroy. Al ser aprobado, se vinculan Mary Inés Rojas, Eduardo Delgado y Martha Pabón.
} 
La Facultad de Educación y la Universidad ganaron un espacio privilegiado al que hoy, además del equipo investigativo, pertenecen otros académicos que, desde sus distintas áreas de formación, desarrollan trabajo docente e investigativo en el intento de comprender el mundo del silencio; asimismo, se han vinculado estudiantes de los diferentes proyectos curriculares que desarrollan trabajos de grado orientados a comprender aspectos disciplinares, pedagógicos y didácticos en relación con la educación de la comunidad sorda.

Con el ingreso de la población sorda a la UPN -segundo semestre de 2003-, la reformulación del proyecto dio lugar al macroproyecto institucional Manos y Pensamiento: Inclusión de estudiantes sordos a la vida universitaria, respuesta social a las necesidades de educación superior de esta comunidad, que propende la formulación de políticas, lineamientos y determinación de acciones pedagógicas con impacto en escenarios locales, regionales y nacionales.

La visión de apertura y reconocimiento a la diversidad, bajo la perspectiva de una universidad para todos, planteó el reto de hacerla accesible a la comunidad sorda, y generó una interesante apuesta de formación pedagógica que, hoy en día, cuenta con una población aproximada de 70 estudiantes sordos, con matrícula vigente en 11 de los 19 programas de licenciatura que ofrece ${ }^{8}$. Sin duda, la formación de educadores para la comunidad sorda -maestros sordos y maestras sordas- permitirá, en cercano futuro, una mejor calidad de vida y participación social con base en el reconocimiento de sus diferencias y la potenciación de sus capacidades lingüísticas, cognitivas y humanas.

El eje del actual proyecto de inclusión universitaria lo constituye el siguiente interrogante: ¿Cómo formar licenciados sordos bilingües que atiendan, con alta calidad, la educación de la comunidad sorda colombiana? Este eje está en consonancia con la misión de la UPN: Formación de educadores en sus distintos programas curriculares, dado el carácter uniprofesional que la distingue de las demás instituciones universitarias.

Este interrogante genera algunos problemas concretos-objeto de permanente reflexión pedagógica y trabajo investigativo- que giran en torno a la potenciación de su primera lengua -lengua de señas-, los métodos de enseñanza de la segunda lengua -castellano lectoescrito-, el papel del intérprete en el proceso enseñanzaaprendizaje y el tipo de adecuaciones curriculares y didácticas pertinentes.

Ciertos interrogantes formulados a partir de la experiencia con los estudiantes sordos condujeron al planteamiento de varios proyectos investigativos, entre los que se destacan dos que han sido aprobados por el Centro de Investigaciones de la Universidad Pedagógica Nacional (CIUP): uno, relacionado con la elaboración de un Estado del arte sobre inclusión de personas sordas a la educación

\footnotetext{
${ }^{8}$ Los estudiantes sordos están matriculados en las siguientes licenciaturas: Educación Física, Biología, Matemáticas, Química, Diseño Tecnológico, Español-Inglés, Ciencias Sociales, Educación Especial, Educación Infantil, Psicología y Pedagogía, y Artes Visuales.
} 
superior, con investigación culminada; y otro, en curso, referido a la construcción de un modelo de inclusión de estudiantes sordos a la educación superior.

Si bien hace una década dudábamos de nuestro papel frente a la educación de los sujetos sordos, podemos decir que el camino recorrido abonó un campo sobre el cual nos movemos actualmente con interés, convicción y decisión, y nos ha posibilitado reconocer en ellos su sensibilidad artística, plástica, estética, axiológica, lúdica, además de su potencial cognitivo y lingüístico.

Conocer la interioridad de quienes por tantos años han estado en el mundo del silencio es interesante apuesta para una universidad cuya misión y sentido es aportar en el desarrollo pleno de la humanidad.

\section{¿Qué fundamentos legales sustentan el proyecto de inclusión de estudiantes sordos a la educación superior?}

La inclusión de estudiantes sordos a la Universidad Pedagógica Nacional está fundamentada en el marco legal y en los principios rectores que guían el tratamiento a la diferencia ${ }^{9}$, en el sistema educativo nacional e internacional. El análisis de estos referentes ha sido esencial para orientar el modelo que, día a día, se reelabora como producto de la inclusión a la vida universitaria.

Aunque la formación de maestros sordos bilingües para atender la población sorda es tendencia reciente en el mundo por cuanto surge, casi un siglo más tarde, después de una larga trayectoria de educación oralista que solamente dejó estancamiento y tristeza en la comunidad sordomuda ${ }^{10}$, producto de los lineamientos impartidos en el Congreso de Milán ${ }^{11}$ (1880), los procesos de integración y, ahora, de inclusión han marcado un importante hito para que las comunidades sordas recuperen los derechos que les fueron vulnerados, entre ellos, la prohibición de usar su primera lengua y la negación de acceder a una educa-

\footnotetext{
${ }^{9}$ Concepto referido por Alcantud (1997, p. 29) para significar el conjunto de estrategias y actuaciones encaminadas a superar todas las dificultades que plantea a la educación la presencia de personas con discapacidad.

${ }^{10}$ Término estigmatizante y en desuso, con el cual los hablantes denominan a quienes, por la sordera, no pueden hablar. Este término surgió por la falsa creencia de que los sordos no pueden adquirir lenguaje.

${ }^{11}$ Realizado en Milán, Italia. En él se impuso la lengua oral como obligatoria en la educación de las personas denominadas en esa época sordomudas debido a que los oyentes allí reunidos, con tradición oralista, simpatizantes de la corriente alemana liderada por Samuel Heinecke, tomaron la decisión de prohibir, a nivel mundial, el uso de la lengua de señas, por considerar que con ella los sordos no podían vincularse a las sociedades oyentes. Este crítico momento debilitó el excelente avance que, en materia educativa, habían logrado los sordos gracias al uso de la lengua manual que les posibilitó desarrollo intelectual y participación ciudadana. Así se produjo estancamiento y tristeza tanto en el desarrollo cultural como en los procesos enseñanza y aprendizaje, tal como la reportan Sacks (2000) y Sánchez (1990).
} 
ción acorde con los excelentes avances alcanzados ${ }^{12}$, en ese entonces, gracias a la influencia de la corriente francesa ${ }^{13}$. Este deterioro específico, en el desarrollo cultural del niño sordo y en la enseñanza y alfabetización de la comunidad sorda, ha sido profundamente estudiado por investigadores como Sacks (2000) y Sánchez (1990).

Debieron pasar casi doscientos años de deprivación del derecho a su lengua (Pineda, 2000) para que los sordos, especialmente los europeos, lograran acceder a una educación bilingüe ${ }^{14}$. Gracias a la aprobación de una ley promulgada por el Parlamento Sueco sobre el reconocimiento de la lengua de señas como lengua de enseñanza, se reiniciará un movimiento en favor de su educación que repercutirá significativamente en distintos países y tomará mayor fuerza con los movimientos mundiales sobre el derecho a la educación de las poblaciones con necesidades especiales. Así, la Declaración Universal de los Derechos Humanos (1948), la Declaración Mundial Educación para Todos (Jomtien, 1990), la Declaración de Viena (1993) y el Programa de Acción adoptado en la Conferencia Mundial de las Naciones Unidas sobre Derechos Humanos (1993), el Marco de Acción sobre Necesidades Educativas Especiales (Salamanca, 1994), las Normas Uniformes sobre la Igualdad de Oportunidades en la Asamblea General de las Naciones Unidas (1993) y el Foro mundial sobre educación para todos (Dakar, 2000) enfatizarán en el derecho a la educación y el compromiso mundial de garantizar el acceso de todos los individuos al sistema educativo, tomando, como referentes, principios relacionados con la equiparación de oportunidades, la no discriminación y la no exclusión de los escenarios educativos, sociales y laborales de las personas con capacidades diferentes.

En Colombia, la huella de esta corriente se hizo palpable a partir de la década de los ochenta cuando toma auge la postura integracionista que plantea, con base en los Derechos Humanos y en la Educación para Todos, la importancia de que las personas con necesidades especiales sean integradas a la educación regular. Esta tendencia mundial, la Carta Política de 1991, la Ley General de Educación (Ley 115 de 1994), su Decreto Reglamentario (2082 de1996), la Ley 324 de 1996 que reconoce la Lengua de Señas colombiana como lengua de la

\footnotetext{
12 Oliver Sacks, en su libro Veo una voz. Viaje al mundo de los sordos, da cuenta de los excelentes logros que en materia educativa, social y cultural habían alcanzado los sordos antes de 1880. Para los interesados, se recomienda la lectura de su obra.

13 Tendencia representada por el francés Abad Michel Lepée quien, hacia 1760, reconoció el uso de la lengua de señas en la educación de las personas sordas. Para profundizar en este tema se recomienda consultar a Skliar (1997), Sánchez (1990), Sacks (2000) y Rodríguez (2004).

${ }^{14}$ Aunque concepto ambivalente, según Anne-Marie de Mejía, el Ministerio de Educación Nacional y el Instituto Nacional de Sordos, en el documento de orientaciones pedagógicas sobre educación bilingüe, re-comprenden esta educación como aquella que se brinda en un entorno lingüístico para hacer posible el acceso y fortalecimiento de la lengua de señas colombiana como primera lengua, y el castellano escrito como segunda.
} 
comunidad sorda, su Decreto Reglamentario (2369 de 1997) sobre educación bilingüe para sordos y servicios de interpretación, la Resolución 1515 de 2000 relacionada con la educación bilingüe para los sordos colombianos y la Ley 982 de 2005 que reconoce a los sordos como parte del patrimonio pluricultural de la Nación, equiparándolos con los pueblos y comunidades indígenas, favorecerán la emergencia de un educador sordo, debidamente preparado desde corrientes critico-sociales que hagan posible su contribución en los cambios socioculturales y que deberán darse por parte de los miembros de las culturas sorda y oyente.

Esta normatividad posibilitó a los sordos acceder a la educación regular en primaria y secundaria. Si bien las acciones se centraron en estos niveles educativos, se descuidó su ingreso a la Educación Superior. Esta omisión se explica, en parte, con la falsa creencia de su incapacidad lingüística y cognitiva que, por fortuna, ha sido superada a medida que se avanza en el conocimiento sobre la naturaleza, potencialidades y posibilidades de las personas sordas. Posterior al proceso de inclusión que inicia la UPN, el Ministerio de Educación Nacional (MEN) y el INSOR promovieron el debate sobre políticas y lineamientos para el acceso a la educación superior de las personas con capacidades diferentes.

\section{¿Qué referentes pedagógicos sustentan este proyecto de acceso a la educación superior de personas sordas?}

Como se expuso en el apartado anterior, el proceso de inclusión de estudiantes sordos a la UPN tiene como referentes los derechos humanos, los derechos de las personas con discapacidad, los lineamientos nacionales e internacionales sobre educación inclusiva y las experiencias latinoamericanas y europeas de educación superior con este tipo de población. Existen también referentes pedagógicos que orientan las acciones afirmativas que se desarrollan en un proceso de educación inclusiva.

En teoría, se sabe que si bien la educación inclusiva es cuestión social y de derechos humanos encaminada a procurar que nadie se sienta privado de constituirse en sujeto (Eglér, 2003), en la práctica aparecen muchos interrogantes sobre la forma como los aspectos consagrados en esas visiones sean asequibles, precisamente por la diversidad del pensamiento, por las especificidades propias de la condición de cada sujeto y las representaciones sociales que tienen los miembros de las culturas mayoritarias hacia las comunidades históricamente excluidas. Ello implica, desde la teoría de la complejidad (Morin, 2002), analizar los múltiples factores que confluyen cuando se educa en el paradigma de la inclusión, porque no basta con situar al sujeto con capacidades diferentes ${ }^{15}$ en

\footnotetext{
${ }^{15}$ Concepto fundamental generado para recomprender los procesos de inclusión desde los enfoques centrados en la valoración del otro como sujeto social, que se constituye como tal en la interrelación permanenete con la cultura.
} 
el escenario educativo, sino que es necesario reflexionar en torno a las distintas dimensiones y disciplinas que se traslapan para asegurar los procesos de transformación en favor de la constitución de un sujeto social.

El caso de la comunidad sorda sirve para ilustrar lo antes mencionado, pues ha sido objeto de exclusión social en distintos momentos de la historia de la humanidad. Sobre ella pesa toda la violencia de las llamadas culturas mayoritarias que, al privilegiar el prosismo ${ }^{16}$, han desconocido el valor de la lengua de señas y negado sus posibilidades como lengua natural, con lo cual los problemas educativos, socioculturales y de desempeño laboral de la comunidad sorda se han desbordado.

Los avances alcanzados en el tema universidad-diversidad por distintos autores, entre ellos Alcantud (1997), Casanova (1997), Asensi (1997), Tolrá (1997), Carbó y Gil (1997), han sido sostén para el proceso de construcción colectiva del modelo de inclusión de estudiantes sordos en la UPN.

En este sentido, Alcantud (1997, p. 34) plantea que los estudiantes considerados en situación de discapacidad deben ser atendidos en forma integral, evitando tratamientos parcelados que ignoren sus diferencias individuales y grupales. Para atender estas consideraciones, el autor propone tres dimensiones que deben tenerse en cuenta: La comunidad universitaria, formada por profesores y el grupo de compañeros; la organización universitaria, que involucra los aspectos académicos administrativos, y, el propio estudiante con capacidades diferentes, que se constituye en el eje central del proceso.

De otra parte, Casanova (1997) plantea la autonomía, la organización flexible, las metodologías variadas y la evaluación formativa como requisitos para el proceso de atención a las poblaciones especiales que acceden al sistema escolar. La autonomía es la capacidad que tiene cada centro universitario o programa académico de organizar el currículo, sin límite de techo, con base en todas las necesidades de las poblaciones especiales que a ellos acuden; la organización flexible, la posibilidad de romper esquemas y tratamientos rígidos para dar vía a las debidas adecuaciones en función de las variables tiempo, espacio, propósitos, estructura curricular, disposición del profesorado, etc.; la metodología supone reconocimiento y respeto al ritmo y estilos de aprendizaje de los estudiantes con capacidades diferentes, lo cual conduce a estrategias que generen mayor interacción tanto con pares como con profesores, y a más participación en los procesos de enseñanza y de aprendizaje, y la evaluación debe asumirse desde la perspectiva formativa donde el descubrimiento y valoración de las dificultades permiten re-significar permanentemente el desarrollo educativo. En términos de Casanova, la evaluación ideográfica; es decir, centrada en cada sujeto, facilita el trabajo sobre posibilidades reales.

\footnotetext{
${ }^{16}$ Término utilizado por Angél Rivière en el prólogo del libro Sistemas alternativos de comunicación, de Sotillo, M. (1999, p. 11), para referir el valor que ha tenido a través de la historia, el habla como ley.
} 
En un proyecto de inclusión de alumnos sordos a la Universidad es necesario tener en cuenta algunas medidas específicas del sistema que, según Casanova, pueden sintetizarse en: refuerzo educativo, adaptación curricular, optatividad y diversificación curricular.

Si bien lo anteriormente expuesto es fundamental para el éxito de los estudiantes con capacidades diferentes en escenarios de educación superior, la permanente orientación académica de ellos también lo es. Asensi (1997) señala la importancia de la orientación vocacional y académica para alcanzar, mediante procesos de reflexión y análisis, un mayor autoconocimiento y ajuste de las capacidades e intereses con opción profesional, y lo que de allí se deriva hacia la futura vinculación sociolaboral; así, el acompañamiento psicopedagógico se hace imprescindible y prioritario.

Otro aspecto esencial para la atención de la diversidad ${ }^{17}$ en la Educación Superior lo constituye el proceso formativo que se realice con la comunidad universitaria. Sobre este tópico, Carbó y Gil (1997) recomiendan desarrollar acciones que conduzcan a la toma de conciencia sobre quiénes son las personas con necesidades especiales, y ello exige un conocimiento previo de las actitudes e imaginarios de todos los miembros de la universidad y la planeación de programas de sensibilización. Exposiciones de fotografía, ciclos de cine temático, circuitos, mesas de coloquio, integración social y cursos formativos son, entre otros, acciones esenciales.

El proceso de inclusión de estudiantes con capacidades diferentes a la vida universitaria requiere de pensar y disponer servicios de apoyo; Girona (1997) sugiere una estructura de acogida, de información y acompañamiento que debe contribuir al desarrollo físico, personal y social, mediante la utilización debida de recursos y servicios, y prestar atención desde el momento en que la persona muestra interés de ingreso a estudios universitarios hasta el de la ubicación laboral; es decir, antes, durante y después de graduarse. Cada centro universitario deberá garantizar acciones que favorezcan la vida estudiantil en todas y cada una de sus dimensiones. Entonces, implica el establecimiento de una política global y la puesta en marcha de medios humanos y financieros en que participen diversos estamentos y agencias de la sociedad. En tal sentido, Tolrá (1997) señala que la política de integración de estudiantes con necesidades especiales a la enseñanza superior debe basarse en ocho principios: universalidad, globalidad, integración, coordinación, igualdad de oportunidades, participación, información y solidaridad.

Según Azevedo (2006), un aporte fundamental en el proceso de inclusión educativa lo constituye el campo de las tecnologías de apoyo a la discapacidad, ya que su filosofía es contribuir en el mejoramiento de la calidad de vida y la re-

\footnotetext{
17 Principio educativo y estrategia de cambio que debe orientar los procesos pedagógicos, donde las desigualdades se convierten en diferencias y no, en motivación para la exclusión.
} 
solución de problemas de adaptación al medio. La tecnología asistente no podría ser totalmente efectiva si no tomara en cuenta las necesidades de las personas con discapacidad, los contextos reales de actuación donde transcurren sus vidas, el análisis de los obstáculos que les presenta el medio y la forma como, desde ese campo de saber, se aporta en la reducción de esas barreras.

Entonces, resulta imprescindible hablar de la importancia de la tecnología para garantizar una mejor accesibilidad, entendida en todas sus formas: desde el acceso físico hasta el acceso a la información. Para ello, es necesario que la Universidad que reciba personas con capacidades diferentes favorezca la adaptación de tecnologías a la naturaleza de la limitación que tenga el estudiante. Será necesario contemplar, además de las adecuaciones físicas, algunas ayudas que permitan el acceso a la información mediante la navegación en la Web y la biblioteca virtual. Ello impone el mejoramiento de los entornos de aprendizaje por cuanto promueven la experimentación y el trabajo personal.

El acceso de estudiantes sordos a la educación superior exige, dada su naturaleza, replanteamientos sustanciales de comunicación y lenguaje, en virtud de que se trasciende de un modelo monolingüe ${ }^{18}$ a otro bilingüe ${ }^{19}$, lo cual constituye un problema que debe ser interpretado desde la transformación de prácticas culturales que no solo se sitúan en la cotidianidad de la vida universitaria, sino que permean el currículo, demandándole cambios significativos en los procesos de enseñanza-aprendizaje.

La relación universidad-diversidad, en tanto tema actual de debate en el ámbito de la educación superior, debe estar presente al formular un modelo de educación inclusiva. Dentro de esta relación, se acogen, para discusión y reflexión, conceptos claves como discapacidad, integración, inclusión, accesibilidad y calidad de vida que, en otros momentos históricos, fueron ajenos a la dinámica de la vida universitaria, pero hoy cobran importancia en el marco de los derechos humanos y del compromiso de las naciones con todos y cada uno de los miembros de la sociedad, lo cual hace prioritario revisar, en cada país, la normatividad existente y la redefinición de políticas educativas que permitan su inclusión en la educación superior.

\section{¿Cómo se asume, en el proyecto Manos y Pensamiento, el concepto educación inclusiva?}

El proyecto ha sido planteado en el marco de una ideología social y educativa que reconoce el derecho a la participación de todas las personas, de acuerdo

\footnotetext{
${ }^{18}$ Utilización preferente de la lengua oral y escrita en la educación del sordo.

${ }^{19}$ Reconocimiento y uso de dos lenguas en la educación del sordo. Coexistencia, en un mismo sujeto, de la lengua de señas de cada país, como lengua natural, y la lengua oral, en su modalidad escrita, como segunda lengua.
} 
con principios universales de diversidad, igualdad y equidad (Moriña, 2004; Arnaíz, 2003, 2004), una manera de contribuir en el establecimiento de formas más solidarias y plurales de convivencia social. Por esta razón, la propuesta educativa formulada armoniza con el nuevo paradigma de conocimiento sobre la naturaleza humana, que revela el valor de la diferencia, independientemente de la condición étnica, de género, discapacidad y contextos culturales y sociales en que conviven los hombres, y la necesidad de propender hacia su pleno desarrollo mediante el fortalecimiento de la interacción permanente a través de redes sociales y de conocimiento. De acuerdo con la filosofía de la educación inclusiva, la propuesta curricular del proyecto Manos y Pensamiento parte del reconocimiento y aceptación de la diferencia, que se convierte en invaluable riqueza para la innovación y el desarrollo pedagógico, por cuanto genera cambios en toda la dinámica universitaria al reconocer a las personas sordas como sujetos que se relacionan con el mundo, mediante otros lenguajes y otras formas de concebir e interpretar la realidad. En este sentido, la diferencia posee valor positivo y enriquecedor para todos (Moriña, 2004).

Se considera que el proyecto debe aportar en la reducción de la brecha social toda vez que ve, en la interacción de los mundos del sordo y del oyente, opciones para el cambio y la construcción de formas más solidarias fundamentadas en el respeto y reconocimiento de la diferencia. Al respecto, Moriña (2004) plantea que la inclusión va más allá del ámbito educativo puesto que permea los distintos escenarios de participación humana y social.

\section{¿Qué proceso se ha seguido en el desarrollo de la experiencia?}

El proyecto, en sus particularidades y especificidades, obedece a un proceso de construcción colectiva en que las "voces acalladas" (Booth, 1998) han sido recuperadas a través de la narrativa, para compartir su historia educativa, sus sueños y la forma de alcanzarlos. Los padres de familia y los maestros de los sordos han aportado a la comprensión del educando con sordera y a la identificación de la mejor forma de contribuir en su proceso de formación profesional. Los profesores de los distintos programas curriculares de la UPN han hecho invaluables aportes, tanto pedagógicos como disciplinares, infundiéndole mayor dinamismo al compromiso social de formar maestros sordos. Por eso, el Modelo de Inclusión de Estudiantes Sordos a la Universidad Pedagógica Nacional se ha desarrollado desde un enfoque de investigación social que privilegia la metodología de investigación-acción participativa.

Cinco dimensiones orientan el proceso de inclusión en la UPN: Pedagógica, Gestión académico-administrativa, Investigativa, Vida universitaria y Proyección social, consideradas esenciales para garantizar el acceso, la permanencia, la titulación y el seguimiento laboral de la población sorda en la Universidad. 


\section{Dimensión pedagógica}

Sus propósitos son generar reflexión en torno al educando sordo, desarrollar acciones pertinentes para adecuar los procesos formativos a sus condiciones particulares y analizar distintas estrategias en pro de la potenciación del desempeño que lo conduzca a alcanzar un rendimiento académico exitoso. Esta dimensión toma en cuenta las siguientes líneas de actuación.

\section{Constitución del equipo académico}

El equipo responsable del proyecto es interdisciplinario y está conformado por profesores de la Facultad de Educación, la mayoría adscritos al Programa de Educación con énfasis en Educación Especial. El grupo mantiene comunicación permanente con profesores e investigadores internos y externos a la Universidad. En su proceso, ha mantenido contacto con expertos internacionales, especialmente de Suecia, Brasil y Argentina, y con la Universidad de Valencia, por sus experiencias en el campo de la inclusión de personas con discapacidad al ámbito universitario; y expertos nacionales: con el Liceo Francés Louis Pasteur, por su experiencia en educación bilingüe; con el Departamento de Antropología de la Universidad Nacional, por la trayectoria en estudios culturales; con el Instituto Nacional de Sordos (INSOR), dado su recorrido en políticas y lineamientos de integración en Colombia y su trabajo en tecnología close caption, y con la Federación Nacional de Sordos de Colombia, por su arduo trabajo en la formación de intérpretes.

Del mismo modo, el equipo ha sometido a debate el proyecto Manos y Pensamiento: Inclusión de estudiantes sordos a la vida universitaria, en distintos escenarios a los que ha sido invitado por el Ministerio de Educación Nacional y universidades públicas y privadas, locales, regionales y nacionales.

\section{Organización del servicio de interpretación}

Para garantizar que los estudiantes sordos se comuniquen en igualdad de condiciones, el proyecto ha organizado el servicio de interpretación, que cuenta con un coordinador y veintitrés intérpretes que se desempeñan en las diferentes actividades académicas, culturales y administrativas propias de la dinámica universitaria.

En el proceso de inclusión ha sido fundamental el papel de los intérpretes; ellos, mediadores entre personas sordas y oyentes, han hecho posible el intercambio de conocimiento, experiencias, conceptos, necesidades, deseos. Su presencia ha permeado toda la dinámica universitaria, especialmente la académica, y facilitado el encuentro del mundo de la persona sorda con el mundo de la persona oyente.

Cabe mencionar que el servicio de interpretación, en el ámbito de la Educación Superior, demanda el replanteamiento de directrices que sobre funciones, 
protocolo y ética han sido establecidas internacionalmente; ello en virtud de que se generan diversas exigencias en su rol que obligan a repensar su papel en el campo educativo. Por tanto, se ha hecho necesario profundizar en su compromiso como mediador cultural, tutor y asesor de los estudiantes sordos, a través del diseño y ejecución de programas de formación y cualificación de intérpretes en contexto pedagógico.

\section{Semestre cero}

Como alternativa para que los estudiantes sordos pudieran ser partícipes activos de toda la dinámica universitaria, se creó el institucionalmente denominado $\mathrm{Se}$ mestre cero, cuyo propósito es orientar y preparar a los estudiantes sordos en lo académico y en la vida universitaria y profesional. Del mismo modo, el Semestre cero es un momento de encuentro con los iguales, que contribuye a la afirmación de su identidad de persona sorda y elevación de su capacidad interactiva, mediante la puesta en escena de su propia lengua. Valores adicionales de este semestre son el fortalecimiento de su identidad frente a la comunidad sorda y el aporte, en términos de su lengua, a la comunidad oyente.

Hoy, las manos -expresión del lenguaje- han logrado penetrar todos los espacios de la Universidad, y evidencian el respeto a la condición del otro y los principios y políticas de inclusión que dinamizan el trabajo pedagógico de la UPN.

El Semestre cero contribuye, en buena parte, no solo a que el estudiante sordo acceda a la Educación Superior, sino que establezca bases firmes para permanecer exitosamente en ella. Por eso, se espera que, al finalizarlo y aprobarlo, presente mejor desarrollo de su lengua de señas, aceptable manejo del castellano lectoescrito, buen nivel de apropiación del sentido que lo pedagógico tiene en su formación como licenciado, excelente comprensión del valor del intérprete en su proceso educativo y ajustado reconocimiento de los trámites y procesos académico-administrativos.

También, se busca que aprenda a vivir en la comunidad universitaria y vaya integrándose de forma activa a equipos académicos, culturales, recreativos y deportivos, por la participación que se le ofrece desde los espacios académicos y demás instancias de la Universidad.

Desde Semestre cero se propicia su asistencia a conferencias, foros, debates y salidas de campo, que le permiten confrontación con los oyentes y mejor conocimiento de los diversos entornos en los que deberá actuar. También, tiene programados espacios para el conocimiento de sí mismo y del contexto familiar y comunitario, a través de cátedras de orientación profesional, vida universitaria y el programa de orientación a estudiantes.

Superado el Semestre cero, el estudiante sordo opta por un proyecto curricular acorde con sus capacidades, habilidades, actitudes y expectativas. El estudiante, además de registrar las asignaturas definidas por el proyecto curricular 
que eligió, debe cursar las asignaturas de los ambientes de comunicación y pedagogía, ofrecidas por la Facultad de Educación para la comunidad sorda, que fortalecerán su formación mediante el trabajo en segunda lengua y en lengua de señas colombiana.

En síntesis, Semestre cero es considerado un período de adaptación del estudiante sordo a la vida universitaria, con propósitos fundamentales -promoción de la identidad con su comunidad sorda, potenciación de la lengua de señas y el castellano lectoescrito, y la exploración vocacional-profesional- que le permitirán una elección de programa de licenciatura acorde con sus motivaciones, intereses y aptitudes. Todo estudiante sordo admitido en la UPN debe cursar y aprobar el Semestre cero, antes de iniciar estudios en los distintos programas curriculares.

\section{Diseño de los ambientes comunicativo y pedagógico}

La inclusión de los estudiantes sordos a la Universidad ha demandado la creación de espacios de formación orientados hacia el fortalecimiento de la lengua de señas, el castellano lectoescrito y el estudio de teorías y prácticas relacionadas con la educación de estas personas, con el propósito no solo de promover la interacción y elevar los niveles de comprensión y conceptualización en las diferentes asignaturas en que participan, sino de enriquecer su formación en la comprensión de procesos de enseñanza-aprendizaje de educandos sordos. Para cumplir con esta finalidad, se diseñaron dos ambientes de formación, comunicativo y pedagógico, ofrecidos y administrados por la Facultad de Educación.

El ambiente de formación comunicativo integra dos espacios académicos: Fortalecimiento de lengua de señas colombiana y Castellano lectoescrito como segunda lengua. Estos espacios académicos, que los estudiantes deben cursar durante diez semestres de la carrera, fueron programados con el fin de potenciar las competencias comunicativas de los estudiantes.

- Fortalecimiento de la lengua de señas como primera lengua. Dado que la lengua de señas no cuenta con códigos suficientes para significar conceptos propios del campo de la pedagogía y de las diferentes disciplinas que abordan en su proceso formativo, se creó un espacio académico denominado Fortalecimiento de la lengua de señas colombiana, desde el cual se busca la generación y consolidación de nuevo vocabulario que contribuya al mejoramiento de los procesos de comunicación y comprensión requeridos para garantizar aprendizajes significativos en los estudiantes sordos.

En sus diferentes niveles, este espacio enfatiza aspectos relacionados con la construcción, discusión, socialización y validación de vocabulario, mediante estrategias de organización y sistematización del mismo, tomando como referente las disciplinas y campos de conocimiento a los que pertenece. También, busca potenciar procesos metacognitivos que permitan al estudiante sordo 
reflexionar sobre su propia lengua. Estos son espacios orientados por modelos lingüísticos; es decir, profesores sordos.

- Fortalecimiento del castellano lectoescrito como segunda lengua. Gran parte de la actividad académica, especialmente en Educación Superior, se encuentra organizada en torno al discurso lectoescrito, una de las formas usuales de circulación del conocimiento que resulta esencial para el aprendizaje y desempeño académico en el mundo de cualquier sociedad letrada. Se espera que los estudiantes sordos alcancen mayor desarrollo en sus procesos de lectura y escritura en una lengua distinta a la natural de ellos -Lengua de señas-; así, se busca que usen la lectura y la escritura como medios de acceso a la información y generación de conocimiento.

A pesar de que la población sorda que ingresa a la UPN viene de un proceso de bilingüismo, el manejo del español es todavía deficiente; por esta razón, el fortalecimiento del castellano está previsto desde Semestre cero hasta décimo semestre. No debe olvidarse que la generación que está accediendo a la universidad ha pasado por una historia muy triste de deprivación de su propia lengua y por un aprendizaje tardío del español, producto de los imaginarios y percepciones que hacia los sordos se tienen socialmente; además, es prudente recordar, sin caer en proteccionismos, que se trata del aprendizaje de un segundo idioma, en su forma lectoescrita, ya que viven en un mundo de silencio carente del input lingüístico con que cuentan los oyentes. Eso hace más complejo el aprendizaje del castellano o de cualquier otro idioma, ya que los sordos deben pasar de una lengua signada, ágrafa, visogestual y no audible, a una segunda lengua, el castellano, con características totalmente distintas: oral, audible, alfabética y escrita. Alegría y Leybaert (1988) y Alegría (1999) señalan que el desconocimiento de la lengua oral es la fuente principal de los problemas de lectura y escritura de los sordos. Vale la pena mencionar los importantes estudios que, en este sentido, han realizado autores como Veyrat y Gallardo (2003), Gutiérrez, R. (2004) y Domínguez y Alonso (2004).

- Ambiente de formación pedagógica. El propósito central de estos espacios es explorar y analizar, de manera crítica y propositiva, los principios y fundamentos pedagógicos que sustentan la educación de personas sordas. A partir de la organización del sistema educativo colombiano, de la función de la escuela, el quehacer del educador sordo y el papel del estudiante, se busca identificar los retos y dificultades que se enfrentan en el ejercicio de la profesión docente. Asimismo, se promueve la reflexión en torno al sentido de la pedagogía y didáctica en el campo de la educación de la comunidad sorda; para tal efecto fueron propuestos, en la Facultad de Educación, dos seminarios de complementación pedagógica que los estudiantes deben tomar en V y VIII semestres. 
Estos espacios académicos, cursados por los estudiantes sordos, independientemente de la licenciatura que hayan escogido, constituyen una oportunidad para reafirmar su identidad como maestros para la población sorda colombiana.

\section{Sensibilización y capacitación de la comunidad universitaria}

El proceso de inclusión de las personas sordas a la UPN ha demandado la implementación de mecanismos de información y comunicación permanentes alrededor de los desarrollos, requerimientos, adaptaciones y expectativas que brotan a diario. El equipo responsable del proyecto desarrolla, de manera sistemática, reuniones en los distintos programas curriculares para analizar el trabajo, las estrategias y necesidades que tienen los estudiantes, y evalúa el desempeño de los intérpretes y los requerimientos del apoyo pedagógico.

Esporádicamente se trabaja con los padres de familia, con el propósito de garantizar el respaldo que ellos deben brindar al proceso educativo y profesional de los estudiantes sordos. Ha sido usual encontrar que el deseo familiar de aportar se ve restringido por la barrera idiomática o por un deficiente manejo de la lengua de señas por parte de los padres. En este tipo de reuniones, la presencia del intérprete es primordial, pues mejora el intercambio de pensamiento y la comunicación sostenida por padres e hijos.

\section{Seguimiento y acompañamiento académico}

El equipo responsable del proyecto se reúne semanalmente para analizar el desarrollo de la experiencia desde sus distintos aspectos. Son momentos para la evaluación de acciones, el análisis de procesos y la toma de decisiones frente a los problemas que surgen en el diario transcurrir, y una oportunidad para conocer las necesidades que se les presenten en los distintos proyectos curriculares.

\section{Apoyo pedagógico y tutorial}

Se ofrece a los estudiantes de acuerdo con sus necesidades académicas. Es parte del proceso pedagógico donde el estudiante tiene la oportunidad de interactuar, fuera de clase, con el profesor de cada uno de los espacios académicos, para solicitar explicaciones sobre temas que le generen dudas o dificultades de comprensión. También en este encuentro, el estudiante sordo goza del acompañamiento del intérprete.

\section{Definición de criterios y estrategias} para la adecuación y ajuste de los planes de estudio

La inclusión de estudiantes sordos a la vida universitaria ha implicado trabajar con la División de Admisiones y Registro, para coordinar aspectos relacionados 
con el proceso de inscripción, pruebas de admisión, publicación de resultados, entrevistas, matrícula, registro de asignaturas, reporte de calificaciones, activación de espacios académicos y transferencia de Semestre cero al proyecto curricular elegido por cada alumno.

Del mismo modo, ha requerido la revisión y análisis de los planes de estudio para definir criterios y estrategias frente a la homologación de asignaturas, el desarrollo de prácticas pedagógicas y el proyecto de grado, aspectos que deben ser definidos con los diferentes programas curriculares, de acuerdo con sus particularidades.

En el caso de las prácticas, se ha concertado que los estudiantes sordos hagan sus intervenciones pedagógicas con población sorda y reciban asesoría no solo desde los aspectos disciplinares, sino desde los teóricos y metodológicos relacionados con la educación de esta población. En la mayoría de los casos, se acude a la estrategia de trabajo colegiado o colaborativo entre los docentes.

Asimismo, se preveen adecuaciones al plan de estudios, en aspectos relacionados con contenidos y actividades pedagógicas, que requieren de un tratamiento diferente, para respetar los estilos de aprendizaje del estudiante sordo. Por ejemplo, si en una asignatura se aborda la enseñanza de la lectura y la escritura en el niño, se incluyen en el programa experiencias y contenidos relacionados con población sorda infantil. En el caso de asignaturas que involucran contenidos y actividades musicales, que no son audibles para ellos, se eligen estrategias que privilegien lo visual y kinestésico.

Cabe destacar que la creación de Semestre Cero y los ambientes de formación comunicativo y pedagógico se ha constituido en alternativa que facilita el ajuste del currículo a la diversidad de los estudiantes.

\section{Evaluación de los aprendizajes}

Este es uno de los aspectos más complejos que suscita inquietudes e interrogantes en el profesorado universitario, debido a que la evaluación de los aprendizajes comúnmente se hace mediante pruebas o trabajos escritos que, para los sordos, significan producir y argumentar en segunda lengua. Como se mencionó anteriormente, la ausencia de input auditivo hace más compleja la comprensión de la sintaxis del castellano que, sumada a la utilización de una lengua visogestual y ágrafa, dificulta la construcción sintáctica en castellano, fenómeno objeto de importantes investigaciones en distintos países.

Ello ha implicado la organización de reuniones en los programas curriculares para analizar las características y dificultades de la evaluación y aportar algunos elementos que contribuyan a la transformación de las prácticas evaluativas de los sordos que, en la mayoría de los casos, deben ser mediadas por los intérpretes y adaptadas a otras modalidades y enfoques que faciliten al profesor la determinación del grado de apropiación de conocimientos y saberes alcanzado por los estudiantes sordos. 
Ha sido necesario insistir, al profesorado, sobre la necesidad de mantener la exigencia académica ya que los sordos deben dar cuenta de la lectura de textos, libros y demás actividades previstas en cada uno de los espacios curriculares; sin embargo, se recomienda, a quienes tienen alumnos sordos, hacer las adaptaciones pertinentes, atendiendo las características particulares de los estudiantes, el grado de dificultad de la materia y la complejidad de los problemas que debe solucionar cada uno de ellos.

\section{Organización y adecuación de medios educativos}

Los estudiantes sordos cuentan, en cada licenciatura, con infraestructura informática conectada a Internet, medios audiovisuales, laboratorios y centros de documentación o bibliotecas satélites, además de la Biblioteca Central. La Facultad de Educación les ofrece, para el trabajo pedagógico, la Sala de Comunicación Aumentativa y Alternativa, dotada de computadores conectados a Internet y adaptados para el desarrollo de actividades pedagógicas, que apoyan el trabajo de algunos espacios académicos como Enseñanza del castellano lectoescrito y Desarrollo del pensamiento lógico matemático, mediante plataforma tecnológica. Se dispone de material audiovisual con vocabulario técnico pedagógico propio de las distintas disciplinas y con software en lengua de señas para el desarrollo de actividades lúdico-pedagógicas.

\section{Dimensión Académico-administrativa}

Está orientada hacia la revisión y propuesta, en el marco de la política institucional, de procesos de gestión y reglamentación que garanticen el pleno cumplimiento del derecho a la educación de todos, y aseguren el ingreso, permanencia y titulación de la población sorda en la universidad.

Dentro de las adecuaciones realizadas cabe mencionar las siguientes.

\section{Proceso de selección de aspirantes, de acuerdo con las condiciones y características de las personas sordas}

La persona sorda que se inscribe a la UPN debe presentar, además de las pruebas establecidas por la Universidad, pruebas específicas de lengua de señas y castellano lectoescrito.

En virtud de que el modelo de inclusión a la UPN parte de un enfoque bilingüe, el proceso de selección toma en cuenta el desempeño del estudiante sordo en lengua de señas colombiana y castellano lectoescrito, con el fin de establecer el nivel expresivo y comprensivo en estos dos idiomas. Es necesario que el sordo sea proficiente en lengua de señas, debido a que en clase recibe los conocimientos en esta lengua, por medio del servicio de interpretación, para luego profundizar en ellos a través de la lectura obligatoria y sugerida de textos y documentos en castellano. 


\section{Adecuación y concertación de procesos administrativos}

Con la División de Admisiones y Registro, se coordinan acciones propias del proceso de inscripción, pruebas de admisión, publicación de resultados, entrevistas, matrícula, registro de asignaturas, reporte de calificaciones y activación de espacios académicos. Adicionalmente se definen procesos y criterios para el registro y transferencia a programas curriculares, legalización académico-administrativa de espacios de formación y adecuación del plan de estudios (en las diferentes licenciaturas donde haya estudiantes sordos).

La Universidad, consecuente con su misión y compromiso social frente al reconocimiento de la diversidad e interculturalidad de sus educandos, ha garantizado la sostenibilidad financiera de este proyecto institucional, con recursos de funcionamiento.

\section{Dimensión Investigativa}

Su objetivo es fomentar la producción y generación de conocimiento en los distintos campos y temas, según el contexto colombiano. Así, los cuestionamientos sobre la pertinencia de métodos para la enseñanza del castellano, el papel pedagógico del intérprete y el tipo de formación requerido por el modelo lingüístico, son ejemplos del sinnúmero de problemas de investigación que ameritan formulación y desarrollo de proyectos investigativos.

Las siguientes son, entonces, líneas de trabajo pedagógico e investigativo: Educación y comunicación, Educación y cognición, Enseñanza de segundas lenguas, Enseñanza de lengua materna, Educación bilingüe, Interpretación en contexto pedagógico, Educación en la diversidad, Universidad-diversidad, Educación inclusiva y Universidad-empleo.

Por el momento, el equipo de Manos y Pensamiento ha adelantado dos proyectos financiados por el Centro de Investigaciones de la Universidad Pedagógica Nacional: Manos y Pensamiento: Proyecto educativo para la población sorda colombiana desde la Universidad Pedagógica Nacional y Construcción de un modelo de inclusión de estudiantes sordos a la Educación Superior.

\section{Dimensión Vida universitaria}

La dimensión Vida universitaria busca garantizar la participación activa de los estudiantes sordos en los distintos escenarios de actuación que ofrece la UPN. A partir del valor que tiene la interacción en el desarrollo de la condición humana, se promueve su vinculación en la dinámica cultural, académica, artística y deportiva, dentro y fuera de la institución.

La vida universitaria del sordo en la UPN, transcurre en las mismas condiciones y oportunidades de los demás estudiantes. Desde el proyecto, se ha generado una importante transformación en la cultura institucional que se refleja 
en la disposición e interés de las distintas Facultades y Divisiones académicas y administrativas por la comprensión de la sordera, del sujeto sordo y su proceso de desarrollo académico.

\section{Dimensión de proyección social}

Pretende fortalecer el diálogo universidad-sociedad, en los niveles nacional e internacional. Mediante proyectos pedagógicos, investigativos, salidas de campo, encuentros con pares, participación en debates, foros, congresos, seminarios, diplomados y cursos de extensión, se busca analizar el impacto que tienen, en los procesos de cambio social, los avances y realizaciones que se derivan del proyecto en cuestión.

La propuesta ha tenido alta incidencia sobre la sociedad, por cuanto ha promovido el acceso a la participación cultural y ciudadana de sujetos a quienes, históricamente, se les ha negado la inclusión social y restringido el disfrute de los bienes y el patrimonio que, en materia de arte, literatura y demás expresiones humanas, ofrece el país. La formación de maestros sordos y maestras sordas confirmará, una vez más, la estrecha relación de interdependencia de los logros académicos, el acceso a la cultura y los ámbitos de participación en la vida ciudadana.

Una significativa repercusión del proyecto tiene que ver con el desarrollo de las prácticas en los distintos escenarios educativos, donde los estudiantes sordos de las diferentes licenciaturas desarrollan acciones formativas con poblaciones de educación inicial y básica.

Otro interesante aporte académico lo constituye el conocimiento que proviene de la interacción que el proyecto ha establecido con distintas Normales del país y algunas instituciones universitarias, para determinar el nivel de desarrollo que en materia de educación inclusiva ha alcanzado Colombia.

La trayectoria en este proyecto ha hecho posible el diseño y elaboración de propuestas formativas en distintos niveles y modalidades -cualificación de intérpretes en contexto pedagógico, lengua de señas, castellano como segunda lengua y modelos de educación inclusiva para estudiantes sordos- y alianzas con distintas instituciones del sector educativo, para desarrollar proyectos conjuntos e implementar prácticas docentes.

\section{Consideraciones finales}

La experiencia vivida deja ver que la inclusión, aunque utopía, es posible; requiere de comunión de voluntades y disposición de ánimo para viajar por rutas inexploradas, con el propósito de descubrir nuevas vías que permitan, a los educandos con capacidades diferentes, lograr la plenitud que desde la pedagogía, históricamente, se ha deseado. Sabemos que ese trasegar complejo e ilimitado depende de nuestra capacidad para soñar y alimentar nuestras convicciones. 
El compromiso social, la participación activa de toda la comunidad universitaria, la reflexión permanente y el estudio sistemático permitirán alcanzar los propósitos y los sueños de una sociedad esperanzada en la cohesión social.

Manos y Pensamiento, avaluada como experiencia exitosa ${ }^{20}$ por el Ministerio de Educación Nacional (MEN), en el año 2007, ha sido objeto de socialización y réplica en algunas instituciones de educación superior ${ }^{21}$ en razón al interés de que la educación superior sea accesible a todos los colombianos. Gracias a este tipo de interlocución, el proyecto ha logrado generar redes interinstitucionales con el propósito de mantener el intercambio de conocimiento.

\footnotetext{
${ }^{20}$ Se habla de programa exitoso debido a que se estructura, desde sus orígenes, con base en lineamientos de política nacional e internacional, para el acceso, permanencia y culminación de estudios de educación superior de la población en situación de discapacidad. Del mismo modo, por ser la institución universitaria que atiende, en la región Iberoamericana, el mayor número de estudiantes sordos incluidos en distintos programas de formación de licenciados, por haber generado transformaciones educativas a partir del diálogo entre las dos comunidades, promovido procesos de recomprensión de la sordera y planteado estrategias y adaptaciones curriculares en el marco de la atención a la diversidad.

${ }^{21}$ Universidades de Antioquia, Valle, Pamplona, Quindío y Distrital Francisco José de Caldas.
} 


\section{Bibliografía}

Alcantud, F. (1997). Universidad y Diversidad. En F. Alcantud (ed.). Universidad y Diversidad (pp. 29-45). Valencia, España: Lliso.

Alegría, J. (1999). La lectura del niño sordo: elementos para una discusión. En A. Domínguez y C. Velasco. Lenguaje escrito y sordera (pp. 59-76). España: Publicaciones Universidad Pontificia Salamanca.

Alegría, J. y Leybaert, J. (1998).

Adquisición de la lectura en el niño sordo. En E. Ochaita y otros. Alumnos con necesidades educativas especiales. Reflexiones sobre educación y desarrollo (pp. 99-121). Madrid: Editorial Popular.

Arnaiz, P. (2003). Educación inclusiva: Una escuela para todos. Málaga, España: Aljibe.

Arnaiz, P. (2004). La educación inclusiva: Dilemas y Desafíos. Revista Educación, Desarrollo y Diversidad, 7 (2), 25-40. Disponible en: http://www. aedes-nacional.com/revista 7(2)articulo2.pdf.

Asensi, B. (1997). Comunicaciones sobre orientación académica de estudiantes con discapacidad. En F. Alcantud (ed.). Universidad y Diversidad (pp. 57-67). Valencia, España: Lliso.

Azevedo, L. (2006). A Model Based Approach to Provide Augmentative Mobility to Severely Disabled Children Through Assistive Technology. Tesis de doctorado para la obtención del título de Doctor en el Departamento de Arquitectura y Tecnología de Computadores, Universidad del País Vasco, Donostia San Sebastián, España.

Bоoтн, T. (1998). El sonido de las voces acalladas: cuestiones acerca del uso de los métodos narrativos con personas con dificultades de aprendizaje. En L. Barton. Discapacidad y sociedad (pp. 253-271). Madrid: Morata.

Casanova, A. (1997). Tratamiento de la diversidad en el sistema educativo actual. En: F. Alcantud (ed.). Universidad y Diversidad (pp. 47-54). Valencia, España: Lliso.

Carbó, B. y Gil, B. (1997). ¿Cómo se puede concienciar la comunidad universitaria? Campaña de sensibilización sobre la problemática de los estudiantes con necesidades educativas especiales. En F. Alcantud (ed.). Universidad y Diversidad (pp. 101-111). Valencia, España: Lliso.

Colombia, Asamblea Nacional ConstiTUYeNTE. (1991). Constitución Política de Colombia. Bogotá: Congreso de la República. Disponible en: http://www. banrep.gov.co/regimen/resoluciones/ cp91.pdf.

Colombia, Congreso de la República. (1996). Ley 324, por la cual se crean algunas normas a favor de la población sorda. Disponible en: http://www.secretariasenado.gov.co/leyes/L0324_96. HTM. 
Colombia, Congreso de la República. (2005). Ley 982, por la cual se establecen normas tendientes a la equiparación de oportunidades para las personas sordas y sordociegas y se dictan otras disposiciones. Disponible en: http://www.secretariasenado.gov.co/ leyes/L0982005.HTM.

Colombia, Ministerio de Educación NACiONAL. (1994). Ley 115, por la cual se expide la Ley General de Educación. Bogotá: MEN. Disponible en: http:// www.mineducacion.gov.co/1621/articles-85906_archivo_pdf.pdf.

Colombia, Ministerio de Educación NaCional. (2000). Resolución 1515, por la cual se establecen los requisitos para la prestación del servicio educativo en el ciclo de educación básica primaria para sordos, por los establecimientos educativos estatales privados. Disponible en: www.insor.gov.co/docs_ insor/Normatividad/resol_1515.doc.

Colombia, Ministerio de Salud, Ministerio de Educación Nacional, Ministerio de ComunicaCiones. (1996). Decreto 2082, por el cual se reglamenta la atención educativa para personas con limitaciones o con capacidades o talentos excepcionales. Disponible en: http://www.presidencia.gov.co/prensa new/decretoslinea/1996/noviembre/18/ dec2082181996.pdf.

Colombia, Ministerio de Salud, Ministerio de EduCACión NaCional. (1997). Decreto 2369, por el cual se reglamenta parcialmente la Ley 324 de 1996. Disponible en: http://www.presidencia. gov.co/prensa_new/decretoslinea/1997/ septiembre/22/dec2369221997.pdf.

Colombia, Ministerio de Educación Nacional, Instituto Nacional de SoRDos. (2006). Educación bilingüe para sordos-etapa escolar-. Orientaciones pedagógicas. Documento 1. Bogotá: Imprenta Nacional de Colombia.

Domínguez, A. y Alonso, P. (2004). La educación de los sordos hoy. Perspectivas y respuestas educativas. Malaga, España: Aljibe.

EgLer, M. (2003). Inclusão Escolar. O que é? Por que? Como fazer? São Paulo: Moderna.

Girona, A. (1997). Los servicios de apoyo para estudiantes con necesidades específicas: una visión europea. En F. Alcantud. (Ed.). Universidad y Diversidad (pp. 145-160). Valencia, España: Lliso.

GutiérRez, R. (2004). Cómo escriben los alumnos sordos. Málaga, España: Aljibe.

MejíA, A. (1998). Educación bilingüe: Consideraciones para programas bilingües en Colombia. Revista El bilingüismo de los sordos, 1 (2), 21-25.

Morin, E. (2002). La noción de sujeto. En D. Schnitman. Nuevos Paradigmas, cultura y subjetividad (pp. 67-85). Buenos Aires: Paidós.

Moriña, A. (2004). Teoría y práctica de la educación inclusiva. Málaga: Aljibe. 
Onu. (1948). Declaración Universal de los Derechos Humanos. Disponible en: http://www.un.org/spanish/aboutun/ hrights.htm.

Onu, Conferencia Mundial Sobre Educación para Todos. (1990). Declaración Mundial sobre educación para todos. Satisfacción de las necesidades básicas de aprendizaje. Jomtien, Tailandia. Disponible en: http://www.unesco. $\mathrm{cl} /$ medios/biblioteca/documentos/ept jomtien_declaracion_mundial.pdf.

Onu, Conferencia Mundial de Derechos Humanos. (1993). Declaración y programa de acción de Viena. Disponible en: http://www.unhchr.ch/ huridocda/huridoca.nsf/(Symbol)/A. CONF.157.23.Sp?OpenDocument.

ONU. (1993). Normas uniformes sobre la igualdad de oportunidades para las personas con discapacidad. Disponible en: http://www.un.org/esa/socdev/ enable/dissres0.htm.

Pineda, R. (2000). El derecho a la lengua. Una historia de la política lingüistica en Colombia. Bogotá: Ediciones Uniandes.

Rodríguez, X. (2004). Una mirada reflexiva hacia el niño sordo. La Habana: Pueblo y educación.

SACKs, O. (2000). Veo una Voz. Viaje al mundo de los sordos. Salamanca: Anaya \& Mario Muchnik.
Skliar, C. (1997). La educación de los sordos una reconstrucción histórica, cognitiva y pedagógica. Argentina: EDIUNC.

SÁncheZ, C. (1990). La increíble y triste historia de la sordera. Caracas: Ceprosord.

Sotillo, M. (1999). Sistemas alternativos de comunicación. España: Trotta.

UnesCo. (1994). Declaración de Salamanca y marco de acción ante las necesidades educativas especiales: París: UNESCO. Disponible en: http://www.unesco.org/ education/pdf/SALAMA_S.PDF.

UnEsCo. (2000). Foro Mundial sobre la educación. Educación para Todos: cumplir nuestros compromisos comunes. Dakar, Senegal. Disponible en: http://www.unesco.org/education/efa/ ed_for_all/dakfram_spa.shtml.

Tolré, J. (1997). PIUNE. Programa de integración de los universitarios con necesidades especiales. En F. Alcantud (ed.). Universidad y Diversidad (pp. 173-177). Valencia, España: Lliso.

Veyrat, M. y Gallardo, B. (ed.) (2003). Estudios lingüísticos sobre la lengua de signos española. Sevilla, España: Publicaciones digitales S.A. 